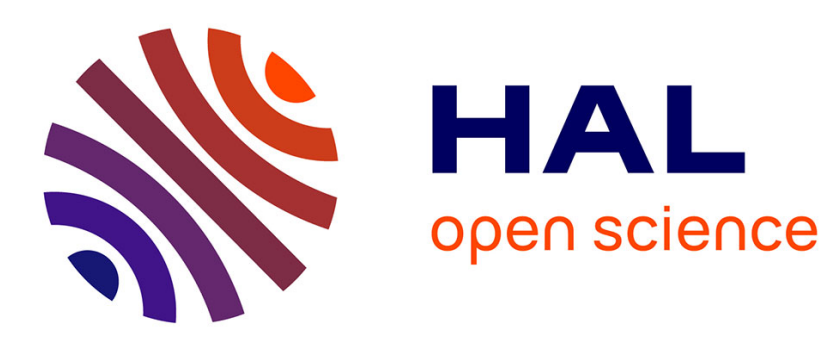

\title{
Les habitats du Bronze final de Portal Vielh à Vendres (Hérault)
}

Laurent Carozza, Albane Burens-Carozza

\section{To cite this version:}

Laurent Carozza, Albane Burens-Carozza. Les habitats du Bronze final de Portal Vielh à Vendres (Hérault). Bulletin de la Société préhistorique française, 2000, 97 (97), pp.573-581. 10.3406/bspf.2000.11165 . hal-00913805

\section{HAL Id: hal-00913805 https://hal.science/hal-00913805}

Submitted on 4 Dec 2013

HAL is a multi-disciplinary open access archive for the deposit and dissemination of scientific research documents, whether they are published or not. The documents may come from teaching and research institutions in France or abroad, or from public or private research centers.
L'archive ouverte pluridisciplinaire HAL, est destinée au dépôt et à la diffusion de documents scientifiques de niveau recherche, publiés ou non, émanant des établissements d'enseignement et de recherche français ou étrangers, des laboratoires publics ou privés. 
Laurent Carozza

Albane Burens

\section{Les habitats du Bronze final de Portal Vielh à Vendres (Hérault)}

In: Bulletin de la Société préhistorique française. 2000, tome 97, N. 4. pp. 573-581.

Citer ce document / Cite this document :

Carozza Laurent, Burens Albane. Les habitats du Bronze final de Portal Vielh à Vendres (Hérault). In: Bulletin de la Société préhistorique française. 2000 , tome 97, N. 4. pp. 573-581.

doi : $10.3406 /$ bspf.2000.11165

http://www.persee.fr/web/revues/home/prescript/article/bspf_0249-7638_2000_num_97_4_11165 


\section{Résumé}

La réalisation d'un lotissement en bordure de l'étang de Vendres, situé dans le département de l'Hérault, a occasionné la fouille d'une portion du site Bronze final du Portai Vielh. Deux campagnes de fouilles, réalisées en 1996 et 1997, ont révélé trois occupations distinctes se rapportant toutes à la fin de l'Age du Bronze. La plus ancienne occupation décelée correspond au Bronze final II : fosses, cuvettes et calages de poteaux ont été découverts associés à un paléosol ponctuellement conservé. L'occupation du Bronze final Illa se caractérise par la présence d'un horizon archéologique en excellent état de conservation, auquel sont associés de nombreux calages de poteaux. A l'exception d'une structure que nous interprétons comme un possible grenier de stockage surélevé, aucun plan cohérent $\mathrm{n}$ 'a pu être dégagé. Au Bronze final Hlb correspond une transformation de I 'espace : creusement d'un fossé d'enceinte, spécialisation du site dans la production de céramiques... L'étude des nombreuses structures d'habitat dégagées sur le gisement permet d'affiner, au travers de la structuration de l'espace et du développement des productions artisanales, notre perception de la dynamique des sociétés du Bronze final.

\section{Abstract}

Due to the building of a new housing estate alongside the Etang de Vendres (Hérault) excavations were carried out on part of the Late Bronze Age site of Portal Vielh. Two excavation campaigns conducted in 1996 and 1997 brought to light three distinct occupation levels, all dating back to the end of the Bronze Age. The earliest corresponds to Late Bronze Age II: pits, post holes and packing material have been discovered together with a palaeo-soil which was well-preserved in places. The Late Bronze Age Ilia settlement is characterised by the good state of preservation of its soil as well as a large quantity of post packing material. It has been impossible to draw any coherent plans except one structure which may have been a raised storehouse. The Late Bronze Age Illb witnessed a change in the area: the digging of a surrounding ditch, specialisation of the site in the production of ceramics... Thanks to the analysis of the numerous dwelling structures discovered on this site, it has been possible, due to the spatial structuring and the development of artisanal productions, to improve our knowledge of Late Bronze Age societies. 


\title{
Les habitats du Bronze final de Portal Vielh \\ Laurent CAROZZA et Albane BURENS à Vendres (Hérault)
}

\begin{abstract}
Résumé
La réalisation d'un lotissement en bordure de l'étang de Vendres, situé dans le département de l'Hérault, a occasionné la fouille d'une portion du site Bronze final du Portal Vielh. Deux campagnes de fouilles, réalisées en 1996 et 1997, ont révélé trois occupations distinctes se rapportant toutes à la fin de l'Âge du Bronze. La plus ancienne occupation décelée correspond au Bronze final II : fosses, cuvettes et calages de poteaux ont été découverts associés à un paléosol ponctuellement conservé. L'occupation du Bronze final IIIa se caractérise par la présence d'un horizon archéologique en excellent état de conservation, auquel sont associés de nombreux calages de poteaux. À l'exception d'une structure que nous interprétons comme un possible grenier de stockage surélevé, aucun plan cohérent n'a pu être dégagé. Au Bronze final IIIb correspond une transformation de l'espace : creusement d'un fossé d'enceinte, spécialisation du site dans la production de céramiques... L'étude des nombreuses structures d'habitat dégagées sur le gisement permet d'affiner, au travers de la structuration de l'espace et du développement des productions artisanales, notre perception de la dynamique des sociétés du Bronze final.
\end{abstract}

\begin{abstract}
Due to the building of a new housing estate alongside the Etang de Vendres (Hérault) excavations were carried out on part of the Late Bronze Age site of Portal Vielh. Two excavation campaigns conducted in 1996 and 1997 brought to light three distinct occupation levels, all dating back to the end of the Bronze Age. The earliest corresponds to Late Bronze Age II: pits, post holes and packing material have been discovered together with a palaeo-soil which was well-preserved in places. The Late Bronze Age IIIa settlement is characterised by the good state of preservation of its soil as well as a large quantity of post packing material. It has been impossible to draw any coherent plans except one structure which may have been a raised storehouse. The Late Bronze Age IIIb witnessed a change in the area: the digging of a surrounding ditch, specialisation of the site in the production of ceramics... Thanks to the analysis of the numerous dwelling structures discovered on this site, it has been possible, due to the spatial structuring and the development of artisanal productions, to improve our knowledge of Late Bronze Age societies.
\end{abstract}

\section{LE SITE}

Le site du Portal Vielh se trouve dans le département de l'Hérault, sur la commune de Vendres. Les habitats de la fin de l'Âge du Bronze sont implantés au nord de l'étang de Vendres, qui forme actuellement une vaste étendue d'eau d'une superficie de 2000 hectares (fig. 1). Le gisement occupe une pointe de terre, témoin de la dépression éolienne à l'origine du creusement de l'étang. Il est bordé, au sud, par un versant abrupt. 


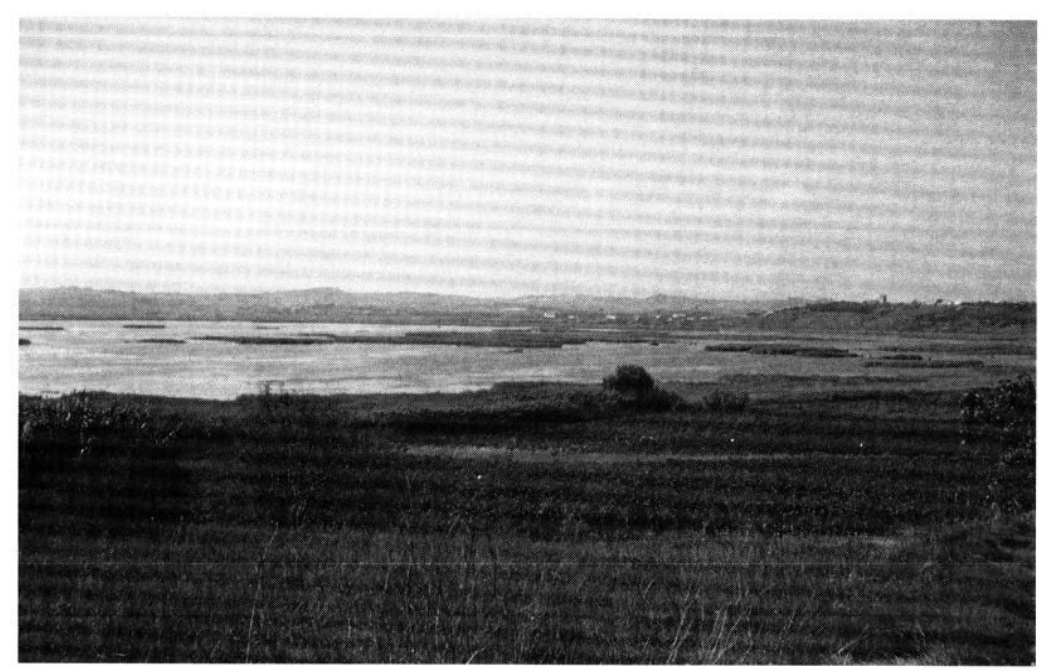

Fig. 1 - Vue de l'étang de Vendres et du versant sud du site de Portal Vielh.

Nous rappellerons que ce site constitue une référence pour le Bronze final languedocien. En effet, dès l'aprèsguerre, les fouilles effectuées par J. Gondar et R. Ross en 1948 avaient permis à ces auteurs de décrire des "fonds de cabanes" et le plan d'unités d'habitation; le paradoxe vient du fait qu'aucun plan de la fouille n'a jamais été publié. La description donnée par les fouilleurs a néanmoins été systématiquement reprise pour illustrer, dans les synthèses régionales, les habitats de plein air. C'est en raison de la construction d'un lotissement que nous avons effectué deux campagnes de fouilles en 1996 et 1997 . Au total, une surface de près de $2000 \mathrm{~m}^{2}$ a pu être décapée. En raison de la nature de l'aménagement, nous avons été contraints de traiter séparément chacun des lots. De ce fait, nous ne possédons qu'une vision partielle de l'occupation du site. De la même manière, les zones qui n'étaient pas touchées par les fondations des bâtiments n'ont fait l'objet que de sondages ponctuels.

L'une des principales difficultés rencontrées lors de la fouille concerne la compréhension de la dynamique de sédimentation. Le site occupant un versant, la mise en place des sédiments a été grandement tributaire de l'érosion de pente. Ainsi, la partie supérieure du site est largement érodée alors que la partie basse, en bordure de l'étang, constitue une zone d'accumulation (fig. 2). Ce phénomène a été amplifié par la structure limonosablonneuse du substratum. Il est à l'origine de la formation, en bas de pente, d'une accumulation sédimentaire. Dans cette zone, les aménagements domestiques disparaissent. Sur une puissance de près de $80 \mathrm{~cm}$, on observe une succession d'horizons homogènes comportant des mobiliers fragmentés. La puissance sédimentaire s'accroît encore lorsque l'on se rapproche de l'ancien rivage.

Dans cette zone, les sondages réalisés par Paul Ambert (UMR 8555 CNRS/EHESS/UPS) ont permis de mettre en lumière l'existence de niveaux de plages. L'examen, par Joël André (Université Paul Valéry, Montpellier), des faunes malacologiques aboutit à la conclusion que nous sommes en présence d'eau salée. Tout porte donc à croire que le cordon littoral n'était pas encore fermé à la fin de l'Âge du Bronze et que la mer (ou une lagune) s'étendait au pied du site protohistorique.

\section{LES OCCUPATIONS DU BRONZE FINAL}

Ce paragraphe est consacré à la succincte présentation de chacune des trois phases d'occupation du site.

\section{Le Bronze final II}

Au Bronze final II, les vestiges se présentent essentiellement sous la forme de creusements. Certaines structures s'apparentent à des petites fosses ou des cuvettes. Ces structures sont souvent comblées de pierres et de fragments de meules en basalte. Le mobilier céramique, bien que morcelé, présente des formes caractéristiques du Bronze final II : décors de cannelures en torsade ou en écharpe. D'autres structures en creux s'apparentent à des silos. Ces derniers sont creusés dans les horizons argilo-graveleux du site. Leur remplissage correspond à des apports successifs, comportant des lentilles cendreuses. Ces lentilles ont permis de recueillir d'abondants carporestes (étude L. Bouby, UMR 8555 CNRS/EHESS/UPS), leur tamisage systématique a également livré de nombreuses mandibules de dorade ainsi que des coquilles de moules et d'huîtres. Les mobiliers associés sont caractéristiques du Bronze final II languedocien.

Le mobilier du silo 19 constitue à cet égard un assemblage modèle composé d'écuelles ornées de cannelures en torsade ou obliques, de jattes à épaulement, ou d'urnes à col (fig. 3).

Les structures sont ponctuellement associées à un paléosol. Ces épandages d'objets sont essentiellement localisés dans la partie médiane du site. Quelques calages de poteaux peuvent avec assurance être rattachés à cette occupation, bien qu'aucun plan de bâtiment ne puisse être présenté. Les concentrations de mobiliers et des structures révèlent une organisation lâche, 

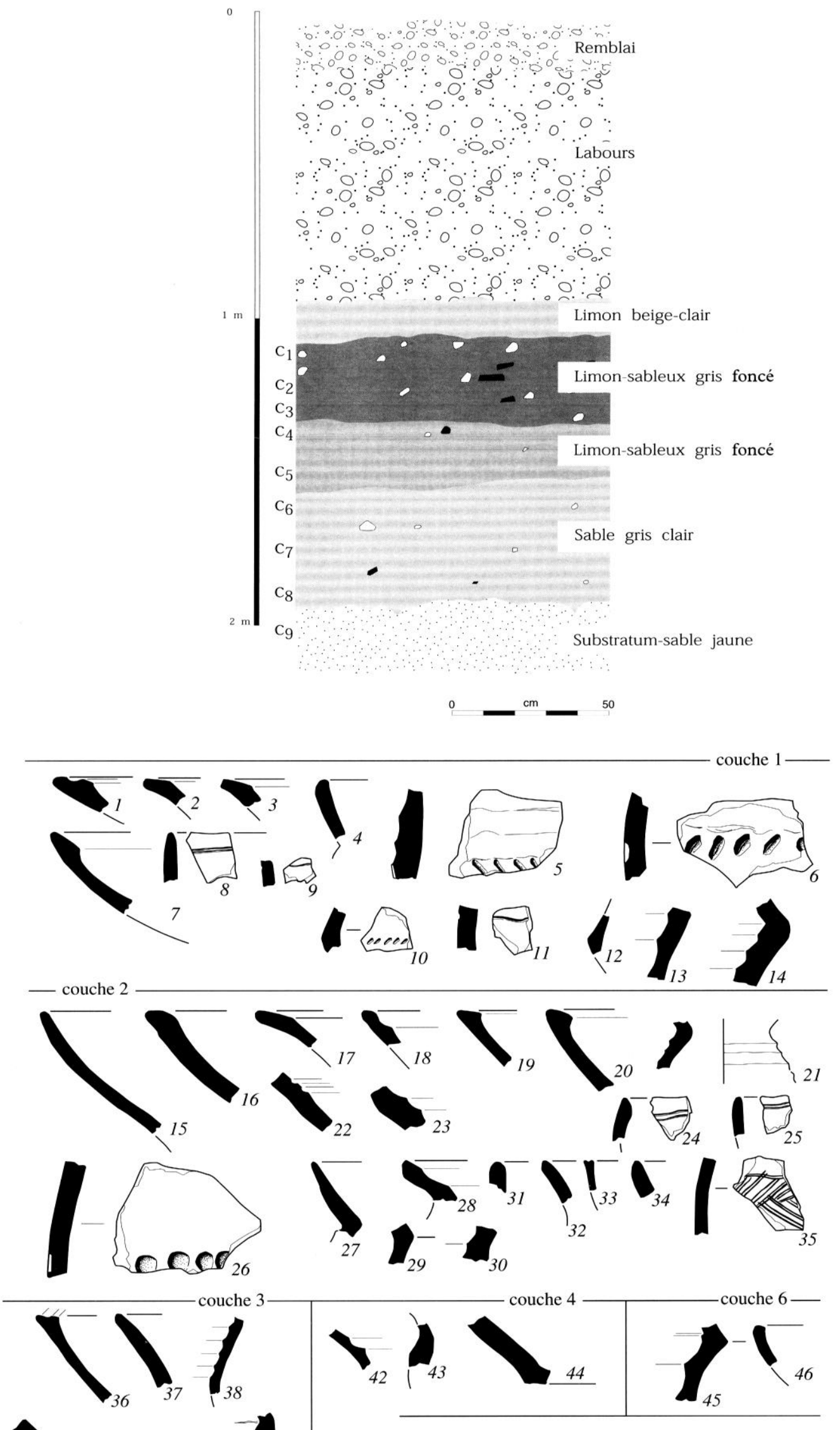

Fig. 2 - Stratigraphie d'un dépôt de bas de pente et mobilier céramique associé. 
comportant de grands espaces vides entre les différents aménagements.

\section{Le Bronze final IIIa}

L'occupation du Bronze final IIIa se caractérise par la présence, sur l'ensemble du site, d'un horizon archéologique dont la mise en place résulte d'apports naturels, par colluvionnement, et d'apports anthropiques. Le bon état de conservation de ces surfaces est illustré par la présence de vases calés dans de petites cuvettes ou de fragments de vases brisés en connexion. De nombreux calages de poteaux sont associés à cet horizon. Si des alignements manifestes sont apparus à
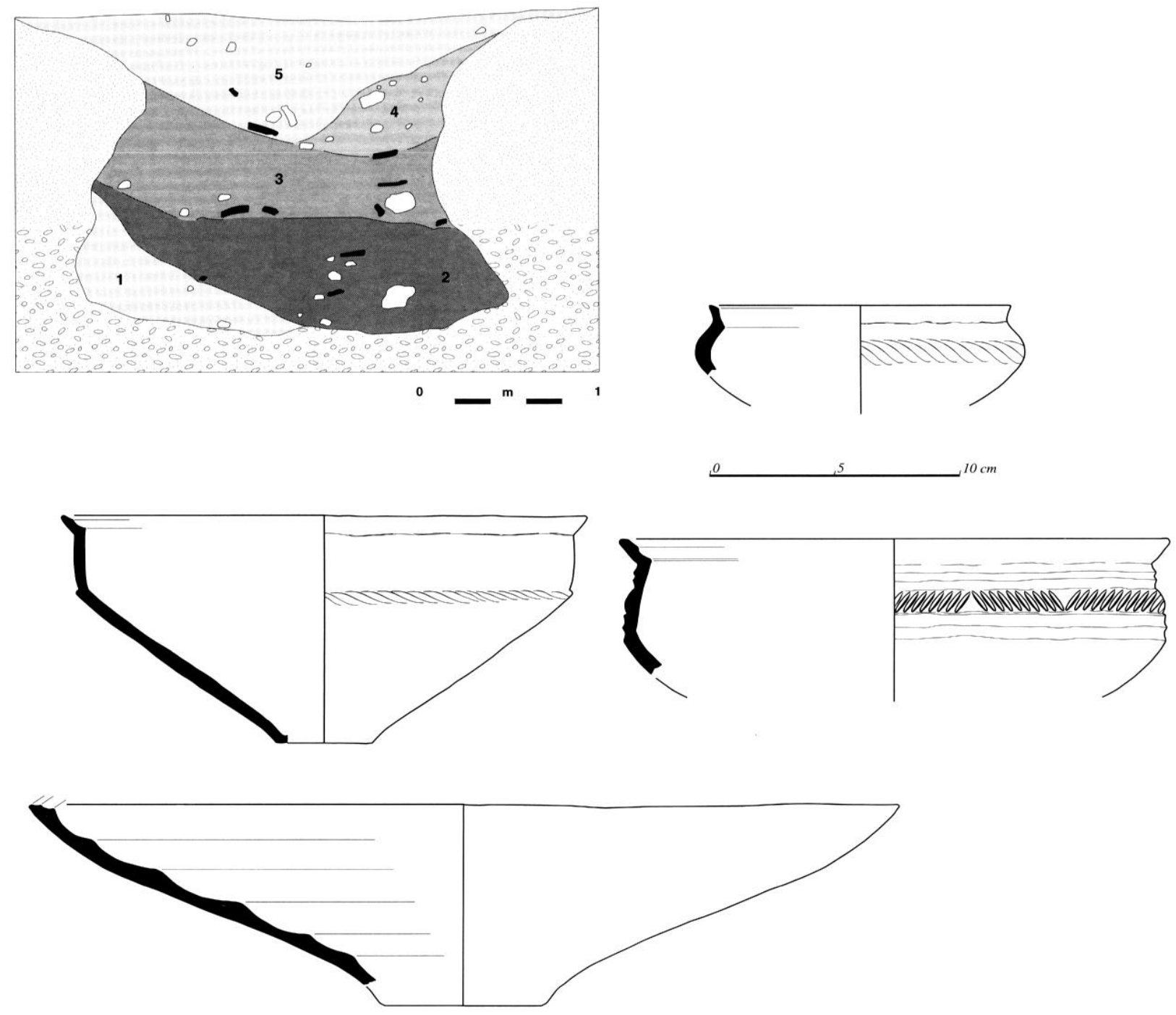

Fig. 3 - Coupe d'un silo daté du Bronze final II et dessin du mobi-

lier céramique issu de son comblement.

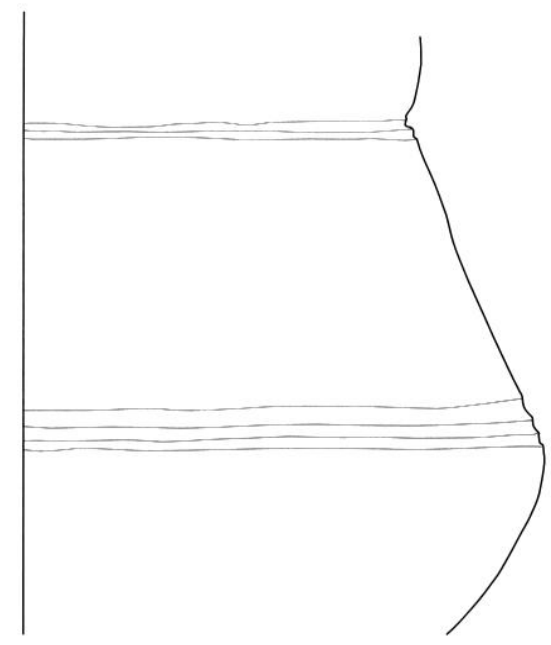




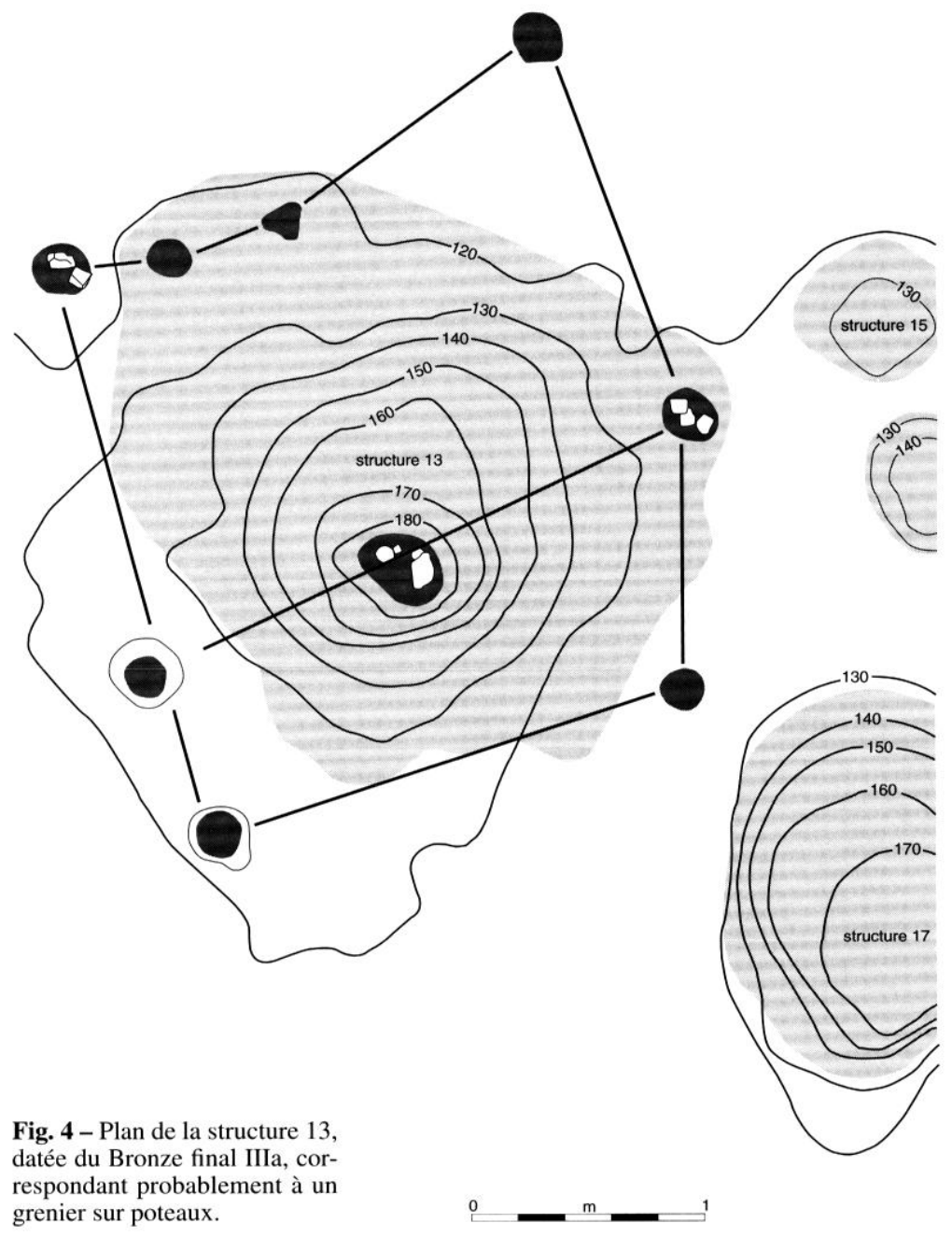

la fouille, il est impossible d'identifier un plan cohérent. La difficulté du repérage des calages de poteaux, creusés dans un horizon sablonneux, rend complexe l'exploitation des plans de fouille. Seules les structures comportant un remplissage nettement différencié de l'encaissant ont pu être identifiées; dans le cas de figure où le poteau ne dispose d'aucun élément de calage, il s'avère impossible de détecter son emplacement.

Si nous ne pouvons pas mettre en évidence le plan des bâtiments, nous disposons néanmoins de structures connexes. Dans la zone argilo-graveleuse du site, des fosses et cuvettes ont pu être dégagées. Il s'agit de fosses d'extraction d'argile dont le creusement s'est interrompu à l'approche des horizons de galets. Leur remplissage inclut de nombreux éléments céramiques.

Une structure plus complexe, que nous interprétons comme un grenier, a pu être identifiée (fig. 4). Il s'agit d'une vaste fosse creusée dans les argiles et qui comporte, à sa périphérie, des trous de poteaux. Au centre de la fosse se trouvait un calage de poteau aux dimensions plus importantes. De plan quadrangulaire, d'une longueur de $3,5 \mathrm{~m}$ pour une largeur de $2 \mathrm{~m}$, cette structure semble correspondre aux modules admis pour les greniers surélevés, l'originalité de ce dernier étant d'être installé sur une fosse. Pour conclure, il appert que les structures qui correspondent à l'occupation du Bronze final IIIa sont réparties sur l'ensemble de l'aire fouillée. Leur densité semble témoigner d'une occupation nettement plus importante que lors de la phase précédente, tant par la nature que par l'extension des vestiges.

\section{Le Bronze final IIIb}

C'est au Bronze final IIIb que l'on constate une nette transformation de l'espace. En amont du site, une structure linéaire en creux correspondant à un fossé a pu être identifiée (fig. 5).

Le fossé présente un creusement en U. Sa largeur est de $5 \mathrm{~m}$; sa profondeur oscille entre 1,4 et $1,6 \mathrm{~m}$. Son remplissage lenticulaire présente la particularité de receler, vers sa base, un horizon comportant de gros blocs calcaires. Certains d'entre eux présentent des modules importants. La présence de ces blocs pourrait être mise en relation avec l'existence d'une petite tranchée, parallèle au creusement du fossé. Nous pouvons envisager que cette tranchée corresponde à l'emplacement d'une palissade, ou qu'il s'agisse d'un élément constituant une fortification. 


\section{hypothèse de restitution}
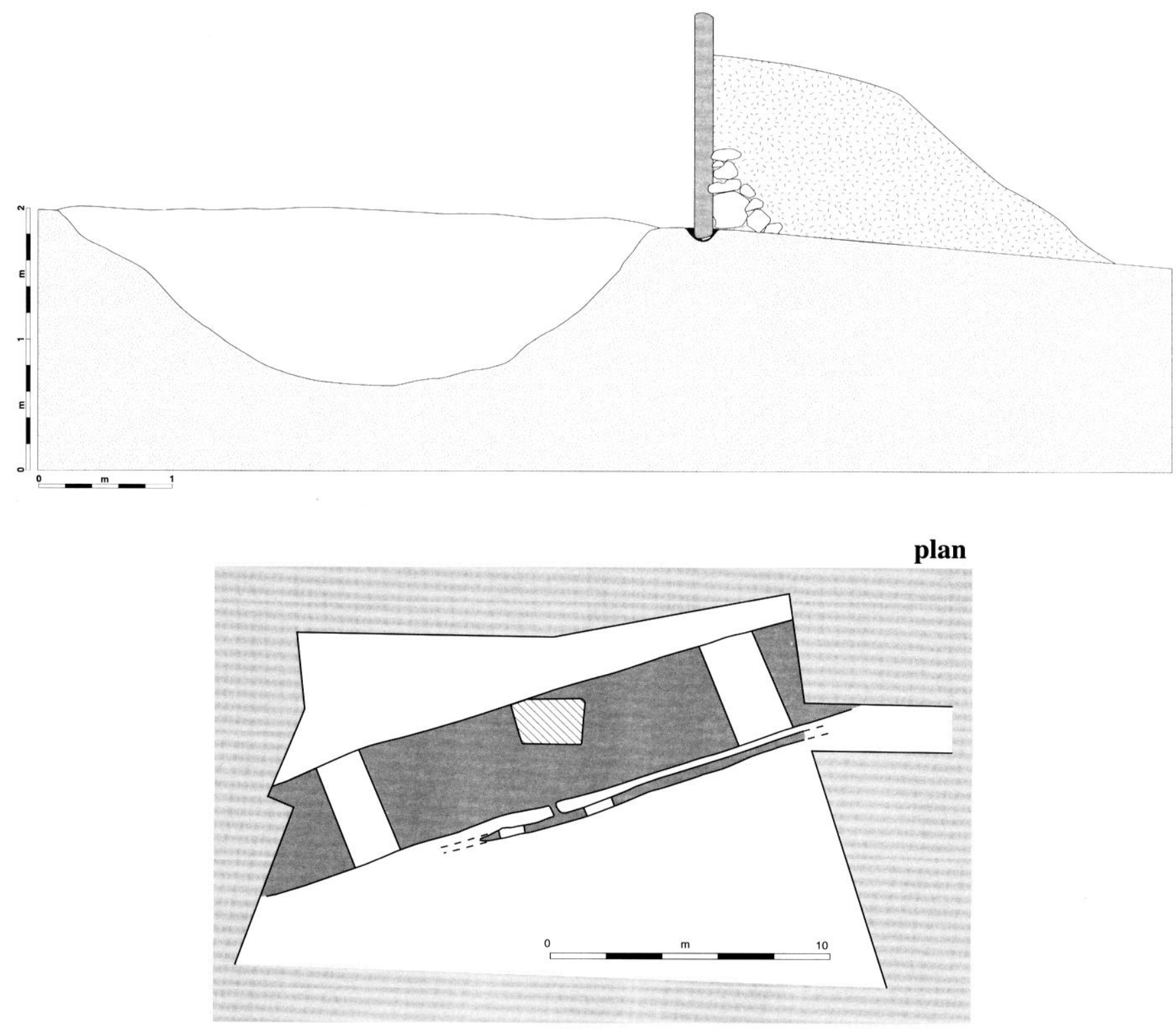

\section{coupe}

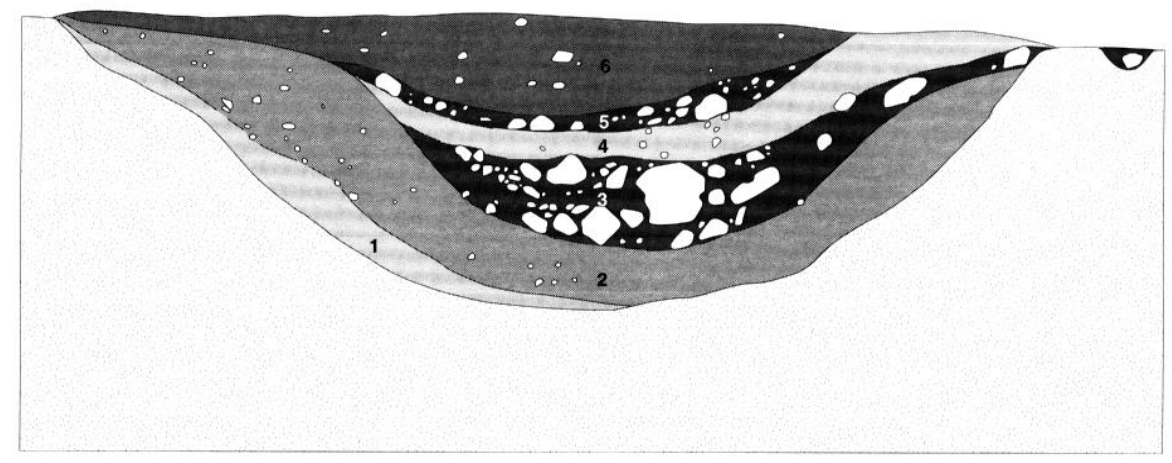

Fig. 5 - Plan, coupe et proposition de restitution de la fortification du Bronze final IIIb du site de Portal Vielh. 


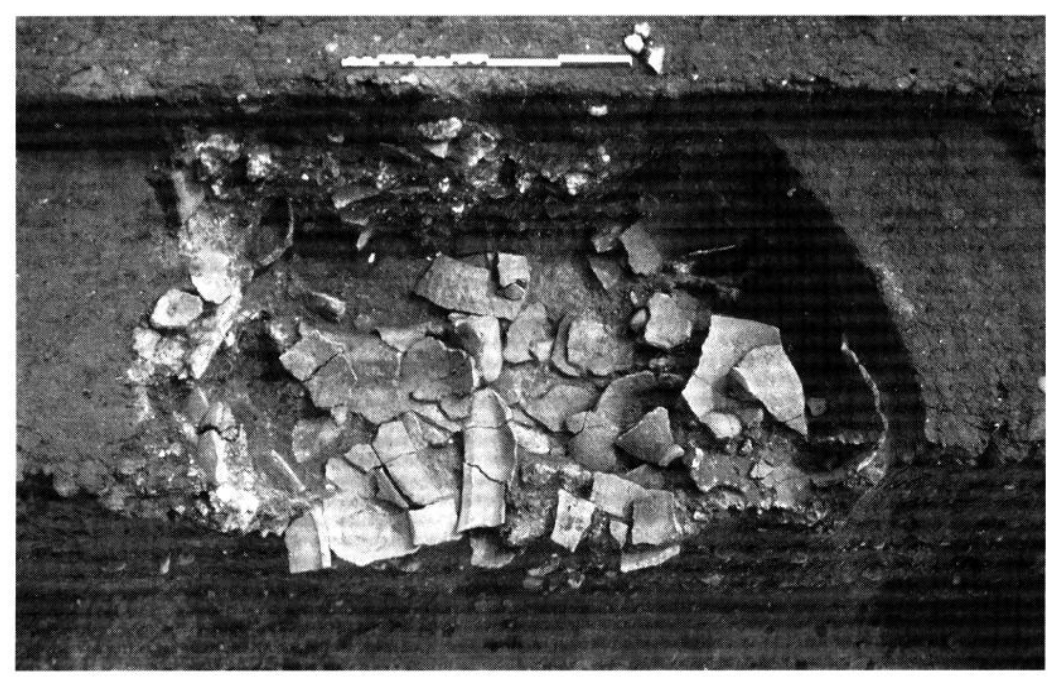

Fig. 6 - Détail du remplissage de la fosse dépotoir ST22. Les vases brisés sont conservés en connexion.

Il serait alors envisageable que des poteaux verticaux soient disposés dans la rigole, alors que les blocs de calcaires assuraient le soubassement d'une levée de terre. Dans ce cas de figure, le site du Portal Vielh serait ceinturé par un véritable rempart de terre et de bois. Les prospections au sol permettent d'avancer l'hypothèse qu'une surface de près de 3 ha était ainsi close. Bien que modeste par sa superficie, en comparaison avec le site de Carsac (Carcassonne, Aude), c'est l'ampleur de la structure défensive qui confère à l'habitat du Portal Vielh un statut particulier.

À l'intérieur de l'enceinte, le paléosol correspondant à l'occupation du Bronze final IIIb a été en grande partie lessivé ou érodé par les travaux agricoles. À l'instar de la phase précédente, des structures en creux ont été mises au jour. On note la présence de nombreux calages de poteaux qui forment des alignements. Néanmoins, aucun plan de bâtiment n'a pu être établi. La présence de soles foyères atteste cependant de l'existence d'unités d'habitation.
Cinq structures en creux particulières ont pu être identifiées. Il s'agit de fosses circulaires, dont le diamètre oscille entre 1,40 et 1,70 m, pour une profondeur d'environ $60 \mathrm{~cm}$. Leur remplissage présente des caractéristiques analogues, car constitué de rebuts de cuisson. Les céramiques issues de ces structures présentent toutes des accidents de cuisson, certaines sont fissurées, d'autres sont fondues (fig. 6). Dans cet amas de céramique, les éléments de terre cuite sont également nombreux. Il s'agit de torchis portant des traces de l'armature de branchage ou de tores circulaires.

Deux structures présentent la particularité de porter des traces de rubéfaction à la base du creusement et sur les parois. La structure 7, d'un diamètre d'environ $1,70 \mathrm{~m}$, est totalement rubéfiée. Son remplissage a livré près de 25 vases différents, tous complets après remontage, mais déformés et surcuits (fig. 7). Les pâtes présentent par endroits d'importants coups de feu et des fissures. Ces récipients sont brisés en connexion et sont mêlés à des tores et des parois de torchis. Les tores sont tous

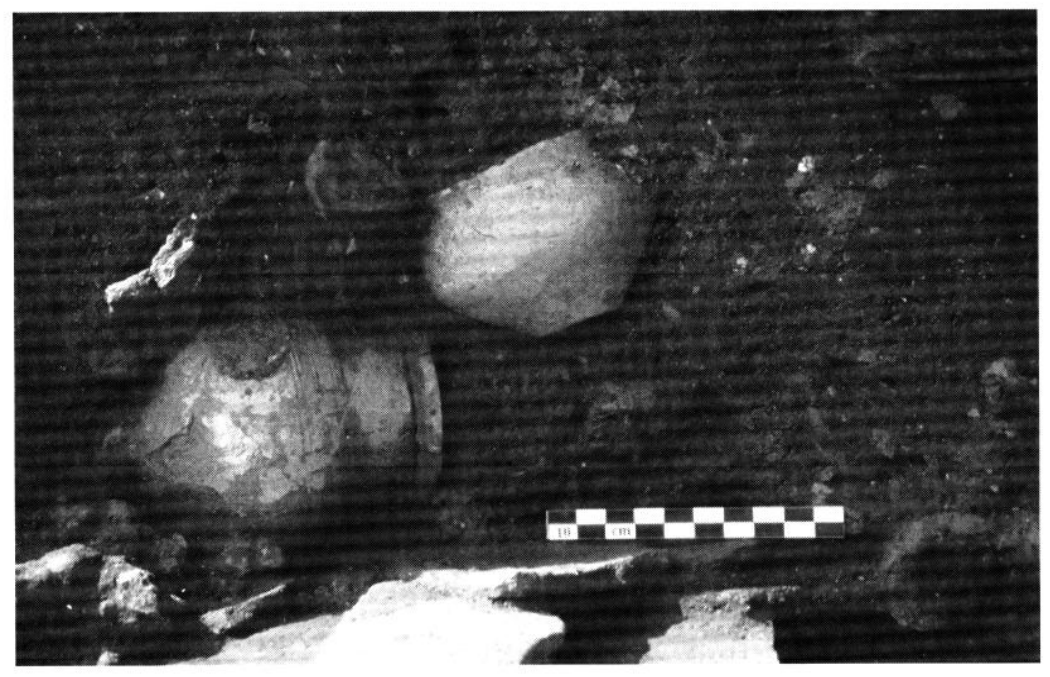

Fig. 7 - Vue de deux gobelets entiers positionnés dans la coupe de la structure 7. 
de même type et présentent des dimensions semblables, environ $20 \mathrm{~cm}$ de diamètre. C'est au sommet du remplissage de la structure 7 que les éléments de terre cuite sur clayonnage étaient les plus nombreux. La structure 57 présente les mêmes caractéristiques : fond et parois rubéfiés. Cette structure a également livré un fragment de sole perforée.

Trois autres fosses possèdent également un remplissage composé de rebuts de cuisson et de fragments de torchis sur clayonnage mais, à l'inverse des structures précédentes, aucune trace de rubéfaction n'a été observée sur les parois des creusements.

Tout porte à croire que ces trois structures correspondent à des fosses dépotoir. À l'inverse, les traces de rubéfaction décelées sur les parois des deux structures précédemment décrites pourraient plaider en faveur de fours, réutilisés comme dépotoirs.

Nous pouvons alors envisager deux hypothèses :

- soit les poteries sont cuites directement dans la fosse probablement recouverte d'une structure en argile sur clayonnage;

- soit les céramiques sont cuites dans un four en cloche.

Cette seconde hypothèse pourrait être étayée par la découverte, au sein du remplissage des structures, de fragments de sole perforée. On peut imaginer un four du même type que celui de Sévrier - en cloche avec une sole perforée - qui reposerait au-dessus de la fosse, faisant office de chambre de combustion. La présence de tores en argile, dont la fonction est de caler les vases et de diffuser la chaleur à l'intérieur des fours, semble accréditer cette hypothèse.

L'analyse en cours des céramiques des dépotoirs va dans le sens d'un assemblage typologique proche entre structures. L'éventail des formes représentées va du gobelet aux grandes jarres. En revanche, les caractères stylistiques de l'ornementation des récipients sont différents. Si la base décorative est constituée de décors incisés au double trait, on peut remarquer des différences importantes. Alors qu'au sein de certaines structures les plats présentent sur leur face interne des décors incisés de type zoomorphe, d'autres n'ont livré que des plats lisses. De la même manière, les écuelles issues de certains dépotoirs possèdent systématiquement des incrustations de matières colorantes, les autres pas. Ces différences notoires pourraient signifier l'existence de différentes officines de production.

La production de céramique semble constituer, sur le site du Portal Vielh, une activité spécialisée. Outre les cinq structures que nous avons fouillées, les prospections réalisées sur le site après un défonçage nous ont permis de détecter plusieurs fosses comportant des rebuts de cuisson. Ces fosses (ou groupes de fosses) sont distantes de plusieurs dizaines de mètres les unes des autres. Notre fouille a d'ailleurs montré que les fosses et les fours sont associés, probablement à proximité d'unités d'habitation.

\section{APPROCHE DIACHRONIQUE \\ DES HABITATS BRONZE FINAL DU PORTAL VIELH}

D'une manière plus générale, et parce qu'il est occupé dans la longue durée, le site du Portal Vielh nous permet d'affiner notre perception de l'économie des sociétés de la fin de l'Âge du Bronze. Tout porte à croire que, dès le Bronze final II et IIIa, nous sommes en présence d'un groupe humain stable qui exploite un territoire composé de terroirs complémentaires. Les plateaux sont propices à l'agriculture alors que les zones calcaires de Lespignan constituent par exemple des zones favorables au pastoralisme. L'ouverture sur la mer est également un fait déterminant dans le choix de l'implantation du site. La pêche ou la collecte de coquillages constitue un apport alimentaire qui, bien que marginal, mérite d'être souligné. De la même manière, la présence de faune sauvage chassée dénote de la diversité des ressources alimentaires. Comme l'indique le creusement de silos ou la construction de grenier, l'agriculture constitue une part importante de l'économie (Bouby et al., 1999).

$\mathrm{Au}$ Bronze final IIIb, le ceinturage du site et l'existence d'une possible fortification témoignent du caractère sédentaire des communautés mailhaciennes du Portal Vielh. La probable spécialisation du site dans la confection de céramiques n'affirme que davantage cette impression.

Le modèle que nous fournit le Portal Vielh est sensiblement différent de celui proposé pour le Languedoc oriental et la zone des lagunes montpelliéraines. Dans cette région, les travaux de Bernard Dedet et Michel Py ont conduit à analyser la société de la fin de l'Âge du Bronze comme semi-sédentaire, ce système se traduisant par des mouvements, cycliques ou saisonniers, entre le littoral et l'arrière pays. Ce modèle nous paraît être en opposition avec les faits observés sur le site du Portal Vielh et, plus généralement, en Languedoc occidental. L'édification d'une fortification, l'existence de stockages en grenier ou en silos attestent la stabilité des communautés humaines. Force est d'admettre que l'édification d'une fortification au Bronze final IIIb suggère une société structurée. L'apparition à cette période de vastes cimetières, souvent situés à proximité des habitats comme c'est le cas sur le site du Portal Vielh, témoigne, avec l'adoption de l'incinération et la nette hiérarchisation de l'habitat, d'une étape fondamentale de l'évolution des sociétés protohistoriques méridionales. Le développement du couple habitat/nécropole et l'apparition de vastes sites fortifiés sont alors le témoignage d'une recomposition du territoire. 


\section{RÉFÉRENCES BIBLIOGRAPHIQUES}

Les références bibliographiques n'apparaissent pas dans le texte selon le souhait des auteurs (ndlr)

BOUBY L., LEROY F., CAROZZA L. (1999) - Food plants from late Bronze Age lagoon sites in Languedoc, southern France : reconstruction of farming economy an environment, Vegetation History and archaebotany, 8, p. 53-69.

CAROZZA L. (1997) - Habitats et cultures à la fin de l'Âge du Bronze en Languedoc et sur le bordure sud-ouest du Massif central, Thèse de Doctorat, École des Hautes Études en Sciences Sociales, Toulouse 1997 , dirigé par J. Guilaine. 3 volumes 398 p. dactylographiées, 418 fig.

CAROZZA L. (sous presse) - Aux sources du premier Âge du Fer languedocien : la fin de l'Âge du Bronze en Languedoc occidental et en Albigeois, in Actes du colloque en hommage à $\mathrm{O}$. et $\mathrm{J}$. Taffanel, septembre 1997.

CAROZZA L., BURENS A. (1995) - Les enceintes protohistoriques de Carsac (Aude) : données nouvelles, Archéologie en Languedoc, $\mathrm{n}^{\circ} 19$, 1996, p. 41-53.

DEDET B. (1985) - Les gisements lagunaires à l'Âge du Bronze Final, in: Occupation des rivages de l'étang de Mauguio, Hérault, au Bronze final et au premier Âge du Fer, éd. ARALO, cahier n 13, p. 5-46.

GONDARD J., ROSS R. (1952) - Trouvailles archéologiques à Béziers et aux environs, Bulletin de la Société d'études Scientifiques de l'Aude, LIII, 1952, p. 217-220.
GUILAINE J. (1972) - L'Âge du Bronze en Languedoc occidental, Roussillon, Ariège, Mémoire de la Société Préhistorique Française, t. 9, 460 p., 134 fig., 11 planches.

PY M. (1984) - Évolution des rapports sociaux de la fin de l'Âge du Bronze à la conquête romaine en Languedoc oriental, in : Archéologie et rapports sociaux en Gaule, Paris, p. 171-183.

PY M. (1990) - Culture, économie et sociétés protohistoriques dans la région Nîmoise, Coll. de l'École Française de Rome, 131, Rome-Paris, 2 vol., 957 p.

PY M. (1993) - Les Gaulois du Midi. Coll. "La mémoire du Temps", éd. Hachette, Paris, 288 p., 50 ill.
Laurent CAROZZA
AFAN/UMR 8555, CNRS/EHESS/UPS
39, allée Jules-Guesde, Toulouse

Albane BURENS

Collège de France/UMR 8555

CNRS/EHESS/UPS

39, allée Jules-Guesde, Toulouse 